\title{
Properties of Balanced Intuitionistic Fuzzy Graphs
}

\author{
M. G. Karunambigai ${ }^{1 *}$, S. Sivasankar ${ }^{2}$ and K. Palanivel ${ }^{3}$ \\ 'Department of Mathematics, Sri Vasavi College, Erode-638016, Tamilnadu, India; \\ karunsvc@yahoo.in \\ 2Department of Science and Humanities, PES University, Bangalore-560085, Karnataka, India; \\ sivshankar@gmail.com \\ ${ }^{3}$ Department of Mathematics, The Oxford College of Engineering, Bangalore-560068, \\ Karnataka, India; sekar4s@gmail.com
}

\begin{abstract}
In this paper, the necessary condition for the Product intuitionistic fuzzy graph to be balanced is given. Some properties and results on the balancing of the regular and product intuitionistic fuzzy graphs are established.
\end{abstract}

Keywords: Balanced IFG, Density of an Intuitionistic Fuzzy Graph (IFG), Product IFG, Regular IFG 2010 Mathematics Subject Classification: 05C72, 03E72, 03 F55.

\section{Introduction}

Intuitionistic fuzzy graphs were introduced by Krassimir T. Atanassov and the operations on intuitionistic fuzzy graphs were defined by Parvathi and Karunambigai in [6]. Balanced intuitionistic fuzzy graphs based on density function were introduced by Karunambigai et al. [3] who analysed the results on direct product, semi strong product and strong product of two intuitionistic fuzzy graphs. Articles [1], [3], [4], [5] motivated us to analyze balanced IFGs and their properties.

This paper deals with the significant properties of balanced intuitionistic fuzzy graphs. The basic definitions and theorem needed are discussed in section 2. Balancing of regular intuitionistic fuzzy graphs and product intuitionistic fuzzy graphs are discussed in section 3.

\section{Preliminaries}

\subsection{Definition [5]}

An intuitionistic fuzzy graph is of the form $G=(V, \mathrm{E})$ where (i) $V=\left\{v_{1}, v_{2}, \ldots v_{n}\right\}$ such that $\mu_{1}: V \rightarrow[0,1]$ and $\gamma_{1}: V \rightarrow$ $[0,1]$, denotes the degree of membership and nonmembership of the element $v_{i} \in V$ respectively and $0 \leq \mu_{1}\left(v_{i}\right)+\gamma_{1}\left(v_{i}\right) \leq 1, \quad \forall v_{i} \in V$,

(ii) $E \subseteq V \times V$ where $\mu_{2}: V \times V \rightarrow[0,1]$ and $\gamma_{2}: V \times V \rightarrow$ $[0,1]$ are such that $\mu_{2}\left(v_{i}, v_{j}\right) \leq \min \left(\mu_{1}\left(v_{i}\right), \mu_{1}\left(v_{j}\right)\right)$ and $\gamma_{2}\left(v_{i}, v_{j}\right) \leq \max \left(\gamma_{1}\left(v_{i}\right), \gamma_{1}\left(v_{j}\right)\right)$, denotes the degree of membership and non-membership of an edge $\left(v_{i}, v_{j}\right) \in E$ respectively, where, $0 \leq \mu_{2}\left(v_{i}, v_{j}\right)+$ $\gamma_{2}\left(v_{i}, v_{j}\right) \leq 1$, for every $\left(v_{i}, v_{j}\right) \in E$.

\subsection{Definition [5]}

An IFG, $H=\left(V^{\prime}, E^{\prime}\right)$ is said to be an IF subgraph (IFSG) of the IFG, $G=(V, E)$ if $V^{\prime} \subseteq V$ and $E^{\prime} \subseteq E$. In other words, if $\mu_{1 i}^{\prime}=\mu_{1 i}, \gamma_{1 i}^{\prime}=\gamma_{1 i}$ and $\mu_{2 i j}^{\prime}=\mu_{2 i j}, \gamma_{2 i j}^{\prime}=\gamma_{2 i j}$ for every $i, j=1,2, \ldots, n$.

\subsection{Definition [6]}

An IFG, $G=(V, E)$ is said to be complete IFG if $\mu_{2 i j}=$ $\min \left(\mu_{1 i}, \mu_{1 j}\right)$ and $\gamma_{2 i j}=\max \left(\gamma_{1 \mathrm{i}}, \gamma_{1 j}\right)$ for every $v_{i}, v_{j} \in V$

${ }^{*}$ Author for correspondence 


\subsection{Definition [6]}

An IFG, $G=(V, E)$ is said to be strong IFG if $\mu_{2 i j}=\min \left(\mu_{1 i}, \mu_{1 j}\right)$ and $\gamma_{2 i j}=\max \left(\gamma_{2 i j}, \gamma_{1 j}\right)$ for every $\left(v_{i}, v_{j}\right) \in E$.

\subsection{Definition [6]}

The complement of an IFG, $G=(V, E)$ is an IFG, $\bar{G}=(\bar{V}, \bar{E})$, where

(i) $\bar{V}=V$,

(ii) $\bar{\mu}_{1 i}=\mu_{1 i}$ and $\bar{\gamma}_{1 i}=\gamma_{1 i}$, for all $i=1,2, \ldots, n$

(iii) $\bar{\mu}_{2 i j}=\min \left(\mu_{1 i}, \mu_{1 j}\right)-\mu_{2 i j}$ and $\bar{\gamma}_{2 i j}=\max \left(\gamma_{1 i}, \gamma_{1 j}\right)-\gamma_{2 i j}$ for all $i, j=1,2, \ldots, n$

\subsection{Definition [3]}

The density of an intuitionistic fuzzy graph $G=(V, E)$ is $D(G)=\left(D_{\mu}(G), D_{\gamma}(G)\right)$ where

$\mu$-density $D_{\mu}(G)$ is defined by

$D_{\mu}(G)=\frac{2 \sum_{u, v \in V}\left(\mu_{2}(\mu, v)\right)}{\sum_{(u, v) \in E}\left(\mu_{1}(u) \wedge \mu_{1}(v)\right)}$, for $u, v \in V$ and

$\gamma$-density $D_{\gamma}(G)$ is defined by

$D_{\gamma}(G)=\frac{2 \sum_{u, v \in V}\left(\gamma_{2}(u, v)\right)}{\sum_{(u, v) \in E}\left(\gamma_{1}(u) \vee \gamma_{1}(v)\right)}, \quad$ for $u, v \in V$.

\subsection{Definition [3]}

An intuitionistic fuzzy graph $G=(V, E)$ is balanced if $D(H) \leq D(G)$, that is, $D_{\mu}(H) \leq D_{\mu}(G), D_{\gamma}(H) \leq D \gamma(G)$ for all subgraphs $\mathrm{H}$ of $\mathrm{G}$.

\subsection{EXAMPLE}

Let $G=(V, E)$ be an intuitionistic fuzzy graph $\mu$-density

$D_{\mu}(G)=2\left(\frac{\begin{array}{c}0.12+0.12+0.08+0.08+0.12+0.08 \\ +0.16+0.08\end{array}}{0.3+0.3+0.2+0.2+0.2+0.3+0.4+0.2}\right)=0.8$

$\gamma$-density

$\mathrm{D}_{\gamma}(\mathrm{G})=2\left(\begin{array}{l}0.51+0.51+0.425+0.425+0.425 \\ 0.6+0.6+0.5+0.5+0.5+0.6+0.6+0.5\end{array}\right)=1.7$

$\mathrm{D}(\mathrm{G})=\left(\mathrm{D}_{\mu}(\mathrm{G}), \mathrm{D}_{\gamma}(\mathrm{G})\right)=(0.8,1.7)$
In Figure 1, for all subgraphs of an IFG $G:(\mu, \gamma, V, E)$ are $\mathrm{H}_{1}:\left(\mu^{\prime} \gamma^{\prime}, \mathrm{V}_{1}, \mathrm{E}_{1}\right), \mathrm{H}_{2}:\left(\mu^{\prime \prime}, \gamma^{\prime \prime}, \mathrm{V}_{2}, \mathrm{E}_{2}\right), \mathrm{H}_{3}\left(\mu^{\prime \prime \prime}, \gamma^{\prime \prime \prime}, \mathrm{V}_{3}, \mathrm{E}_{3}\right)$ such that $V_{1}=\left\{\mathrm{v}_{1}, \mathrm{v}_{2}\right\}, E_{1}=\left\{\left(\mathrm{v}_{1}, \mathrm{v}_{2}\right)\right\}, V_{2}=\left\{\mathrm{v}_{2}, \mathrm{v}_{3}, \mathrm{v}_{4}\right\}$, $E_{2}=\left\{\left(\mathrm{v}_{2}, \mathrm{v}_{3}\right),\left(\mathrm{v}_{3}, \mathrm{v}_{4}\right),\left(\mathrm{v}_{2}, \mathrm{v}_{4}\right)\right\}, \quad V_{3}=\left\{\mathrm{v}_{1}, \mathrm{v}_{2}, \mathrm{v}_{3}, \mathrm{v}_{4}, \mathrm{v}_{5}\right\}$, $E_{3}=\{\}$ be a non empty subgraphs of $\mathrm{G}$.

Density $\left(\mathrm{D}_{\mu}(\mathrm{H}), \mathrm{D}_{\gamma}(\mathrm{H})\right)$ is $\mathrm{D}\left(\mathrm{H}_{1}\right)=(0.8,1.7), \mathrm{D}\left(\mathrm{H}_{2}\right)$ $=(0.8,1.7), \mathrm{D}\left(\mathrm{H}_{3}\right)=(0,0)$

Similarly, $D(H) \leq D(G)$ for all subgraphs $H$ of $G$. Hence $\mathrm{G}$ is balanced IFG.

\subsection{Definition [2]}

The neighbour degree of vertex is defined as $d_{N}(v)=\left(d_{N \mu}(v), d_{N \gamma}(v)\right)$ where $d_{N \mu}(v)=\sum_{w \in N(v)} \mu_{1}(w)$ and $d_{N \gamma}(v)=\sum_{w \in N(v)} \gamma_{1}(w)$.

\subsection{Definition [2]}

The closed neighbour degree of vertex $\mathrm{v}$ is defined as $d_{N}[v]=\left(d_{N \mu}[v], d_{N \gamma}[v]\right)$ where $d_{N \mu}[v]=\sum_{w \in N(v)} \mu_{1}(w)+$ $\mu_{1}(v)$ and $d_{N \gamma}[v]=\sum_{w \in N(v)} \gamma_{1}(w)+\gamma_{1}(v)$.

\subsection{Definition [2]}

The minimum closed neighbour degree is defined as $\delta_{N}[G]=\left(\delta_{N \mu}[G], \delta_{N \gamma}[G]\right)$ where $\delta_{N \mu}[G]=\wedge d_{N \mu}[v]: v \in V$ and $\delta_{N \gamma}[G]=\wedge d_{N \gamma}[v]: v \in V$.

\subsection{Definition [2]}

The maximum closed neighbour degree is defined as $\Delta_{N}[G]=\left(\Delta_{N \mu}[G], \Delta_{N Y}[G]\right)$ where $\Delta_{N \mu}[G]=V d_{N \mu}[v]: v \in V$ and $\Delta_{N \gamma}[G]=V d_{N \gamma}[v]: v \in V$.

\subsection{Definition [2]}

An intuitionistic fuzzy graph $G=(V, E)$ is said to be regular IFG if all the vertices have the same closed neighbour degree. (ie) $\delta_{N \mu}[G]=\Delta_{N \mu}[G]$ and $\delta_{N \gamma}[G]=\Delta_{N \gamma}[G]$ (See Figure 2).

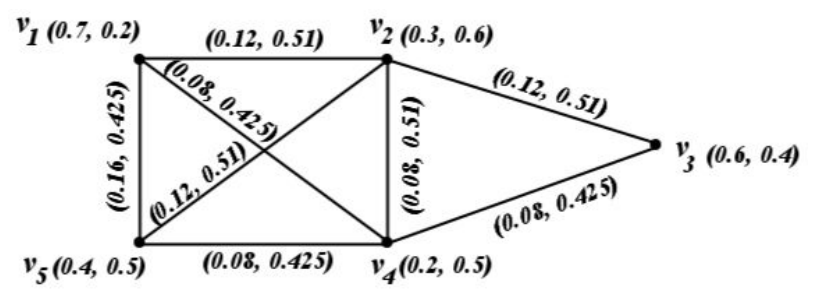

Figure 1. Balanced intuitionistic fuzzy graph. 

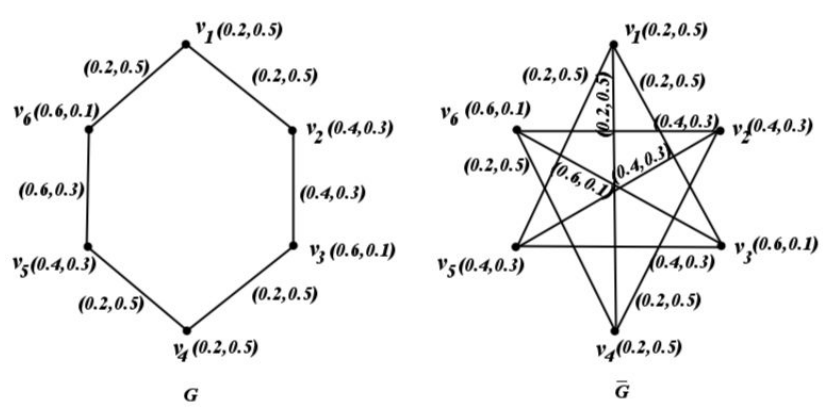

Figure 2. Regular IFG and its complement.

\subsection{Definition [4]}

An intuitionistic fuzzy graph $(G=(V, E)$ is said to product IFG if $\mu_{2}\left(v_{i}, v_{j}\right) \leq\left(\mu_{1}\left(v_{1}\right) \cdot \mu_{1}\left(v_{j}\right)\right)$ and $\gamma_{2}\left(v_{i}, v_{j}\right)$ $\leq \gamma_{1}\left(v_{i}\right) \cdot \gamma_{1}\left(v_{j}\right)$, for every $\left(v_{i}, v_{j}\right) \in E$.

\subsection{Definition [4]}

A product intuitionistic fuzzy graph $G=(V, E)$ is said to complete product IFG if $\mu_{2}\left(v_{i}, v_{j}\right)=\left(\mu_{1}\left(v_{i}\right) \cdot \mu_{1}\left(v_{j}\right)\right)$ and $\gamma_{2}\left(v_{i}, v_{j}\right)=\left(\gamma_{1}\left(v_{i}\right) \cdot \gamma_{1}\left(v_{j}\right)\right)$, for every $v_{i}, v_{j} \in V$.

\subsection{Definition [4]}

A product intuitionistic fuzzy graph $G=(V, E)$ is said to strong product IFG if $\mu_{2}\left(v_{i}, v_{j}\right)=\mu_{1}\left(v_{i}\right) \cdot \mu_{1}\left(v_{j}\right)$ and $\gamma_{2}\left(v_{1}, v_{j}\right)=\left(\gamma_{1}\left(v_{i}\right) \cdot \gamma_{1}\left(v_{j}\right)\right)$, for every $\left(v_{1}, v_{j}\right) \in E$.

\subsection{Definition [4]}

A product intuitionistic fuzzy graph $G=(V, E)$ is said to complement product IFG if $\bar{G}=(\bar{V}, \bar{E})$, where

(i) $\bar{V}=V$,

(ii) $\bar{\mu}_{1 i}=\mu_{1 i}$ and $\bar{\gamma}_{1 i}=\gamma_{1 i}$, for all $i=1,2, \ldots n$

(iii) $\bar{\mu}_{2 i j}=\left(\mu_{1 i} \cdot \mu_{1 j}\right)-\mu_{2 i j}$ and $\bar{\gamma}_{2 i j}=\left(\gamma_{1 i} \cdot \gamma_{1 j}\right)-\gamma_{2 i j}$, for all $i, j=1,2, \ldots n$

\subsection{TheOREM [3]}

Every complete intuitionistic fuzzy graph is balanced.

\subsection{Corollary [3]}

Every strong IFG is balanced.

\section{Properties of Balanced IFG}

\subsection{Definition}

The density of a product intuitionistic fuzzy graph $G=(V, E)$ is $D(G)=D_{\mu}(G), D_{\gamma}(G)$, where $D_{\mu}(G)$ is defined by

$$
\begin{aligned}
& D_{\mu}(G)=\frac{2 \sum_{u, v \in V}\left(\mu_{2}(u, v)\right)}{\sum_{(u, v) \in E}\left(\mu_{1}(u) \cdot \mu_{1}(v)\right)}, \text { for } u, v \in V \text { and } \\
& D_{\gamma}(G) \text { is defined by } \\
& D_{\gamma}(G)=\frac{2 \sum_{u, v \in V}\left(\gamma_{2}(u, v)\right)}{\sum_{(u, v) \in E}\left(\gamma_{1}(u) \cdot \gamma_{1}(v)\right)}, \quad \text { for } u, v \in V .
\end{aligned}
$$

\subsection{Definition}

A product intuitionistic fuzzy graph $G=(V, E)$ is balanced if $D(H) \leq D(G)$, that is, $D_{\mu}(H) \leq D_{\mu}(G), D_{\gamma}(H) \leq D_{\gamma}(G)$ for all sub graphs $\mathrm{H}$ of $\mathrm{G}$.

\subsection{ExAmple}

Consider $G=(V, E)$ be an intuitionistic fuzzy graph as in Figure 3.

$\mu$-density $D_{\mu}(G)=2\left(\frac{0.24+0.16+0.1+0.15+0.4}{0.24+0.16+0.1+0.15+0.4}\right)=2$

$\gamma$-density $D_{\gamma}(G)=2\left(\frac{0.12+0.16+0.32+0.24+0.08}{0.12+0.16+0.32+0.24+0.08}\right)=2$

$D(G)=\left(D_{\mu}(G), D_{\gamma}(G)\right)=(2,2)$

\subsection{Definition}

A product intuitionistic fuzzy graph $G=(V, E)$ is said to be regular product IFG if all the vertices have the same closed neighbour degree. (ie) $\delta_{N \mu}[G]=\Delta_{N \mu}[G]$ and $\delta_{N \gamma}[G]=\Delta_{N \gamma}[G]$.

\subsection{TheOREM}

Every regular IFG is balanced.

\section{Proof:}

Every regular IFG is a strong IFG then by Corollary 2.19, every regular IFG is balanced.

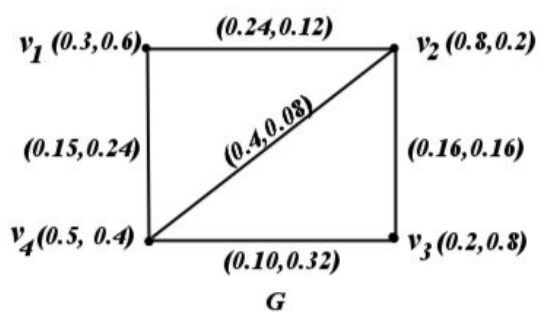

Figure 3. Product intuitionistic fuzzy graph. 


\subsection{THEOREM}

The complement of a regular IFG is balanced.

\section{Proof:}

Let $G=(V, E)$ be a regular IFG and $\bar{G}=(\bar{V}, \bar{E})$ be its complement.

Since G is regular, $\mu_{2}(u, v)=\mu_{1}(u) \wedge \mu_{1}(v)$ and $\gamma_{2}(u, v)=$ $\gamma_{1}(u) \vee \gamma_{1}(v) \rightarrow(1)$ for every $(u, v) \in E$.

In $\bar{G}, \overline{\mu_{2}(u, v)}=\mu_{1}(u) \wedge \mu_{1}(v)-\mu_{2}(u, v)$ and $\overline{\gamma_{2}(u, v)}=$ $\gamma_{1}(u) \vee \gamma_{1}(v)-\gamma_{2}(u, v)$.

Since $G$ is regular, by (1) we have $\overline{\mu_{2}(u, v)}=0$ and $\overline{\gamma_{2}(u, v)}=0$ forevery $(u, v) \in E$ and $\overline{\mu_{2}(u, v)}=\mu_{1}(u) \wedge \mu_{1}(v)$ and $\overline{\gamma_{2}(u, v)}=\gamma_{1}(u) \vee \gamma_{1}(v)$ for every $(u, v) \in \bar{E} \Rightarrow \bar{G}$ is a strong IFG.

Then by Corollary 2.19, $\bar{G}$ balanced.

\subsection{TheOREM}

Every complete product intuitionistic fuzzy graph is balanced.

\section{Proof:}

Let $G=(V, E)$ be a complete product IFG, then by the definition of complete product IFG, we have $\mu_{2}(u, v)=\mu_{1}(u) \cdot \mu_{1}(v)$ and $\gamma_{2}(u, v)=\gamma_{1}(u) \cdot \gamma_{1}(v)$, for every $u, v \in V$.

$$
\begin{aligned}
& \sum_{u, v \in V}\left(\mu_{2}(u, v)\right)=\sum_{(u, v) \in E}\left(\mu_{1}(u) \cdot \mu_{1}(v)\right) \quad \text { and } \\
& \sum_{u, v \in V}\left(\gamma_{2}(u, v)\right)=\sum_{(u, v) \in E}\left(\gamma_{1}(u) \cdot \gamma_{1}(v)\right)
\end{aligned}
$$

Now
$D(G)=\left(\frac{2 \sum_{u, v \in V}\left(\mu_{2}(u, v)\right)}{\sum_{(u, v) \in E}\left(\mu_{1}(u) \cdot \mu_{1}(v)\right)}, \frac{2 \sum_{u, v \in V}\left(\gamma_{2}(u, v)\right)}{\sum_{(u, v) \in E}\left(\gamma_{1}(u) \cdot \gamma_{1}(v)\right)}\right)$
$D(G)=2\left(\frac{\sum_{u, v \in V}\left(\mu_{1}(u) \cdot \mu_{1}(v)\right)}{\sum_{(u, v) \in E}\left(\mu_{1}(u) \cdot \mu_{1}(v)\right)}, \frac{\sum_{u, v \in V}\left(\gamma_{1}(u) \cdot \gamma_{1}(v)\right)}{\sum_{(u, v) \in E}\left(\gamma_{1}(u) \cdot \gamma_{1}(v)\right)}\right)$

$D(G)=(2,2)$

Let $\mathrm{H}$ be a non empty subgraph of $\mathrm{G}$ then, $D(H)=(2,2)$ for every $H \subseteq G$.

Thus $\mathrm{G}$ is balanced.

\subsection{COROLlary}

A necessary condition for the product intuitionistic fuzzy graph is balanced if it is strong.

\subsection{Note}

The converse of the above corollary is need not be true. Every balanced product intuitionistic fuzzy graph need not be strong.

\subsection{EXAMPLE}

Let $G=(V, E)$ be a product intuitionistic fuzzy graph $\mu$-density

$$
\begin{aligned}
D_{\mu}(G)=2\left(\frac{0.13+0.13+0.104+0.104+0.052}{0.2+0.2+0.16+0.16+0.08+0.08}\right. \\
\left.\frac{+0.052+0.039+0.0975+0.078}{+0.06+0.15+0.12}\right)=1.3
\end{aligned}
$$

$\gamma$-density

$$
\begin{gathered}
D_{\gamma}(G)=2\left(\frac{0.108+0.108+0.135+0.135+0.189}{0.24+0.24+0.3+0.3+0.42+0.35}\right. \\
\left.\frac{+0.1575+0.189+0.108+0.162}{+0.42+0.24+0.36}\right)=0.9 \\
D(G)=\left(D_{\mu}(G), D_{\gamma}(G)\right)=(1.3,0.9)
\end{gathered}
$$

In Figure 4, For all subgraphs $\mathrm{H}$ of a PIFG $\mathrm{G}$, $D(H) \leq D(G)$. So $G$ is balanced product IFG. From the above graph easy to see that:

$\mu_{2}\left(v_{i}, v_{j}\right) \neq\left(\mu_{1}\left(v_{i}\right) \cdot \mu_{1}\left(v_{j}\right)\right)$ and $\gamma_{2}\left(v_{i}, v_{j}\right) \neq\left(\gamma_{1}\left(v_{i}\right) \cdot \gamma_{1}\left(v_{j}\right)\right)$, $\left(v_{i}, v_{j}\right) \in E$. Hence $\mathrm{G}$ is balanced product intuitionistic fuzzy graph but not strong.

\subsection{THEOREM}

A complement of a strong product intuitionistic fuzzy graph is balanced.

\section{Proof:}

Let $G=(V, E)$ be a strong product IFG and $\bar{G}=(\bar{V}, \bar{E})$ be its complement.

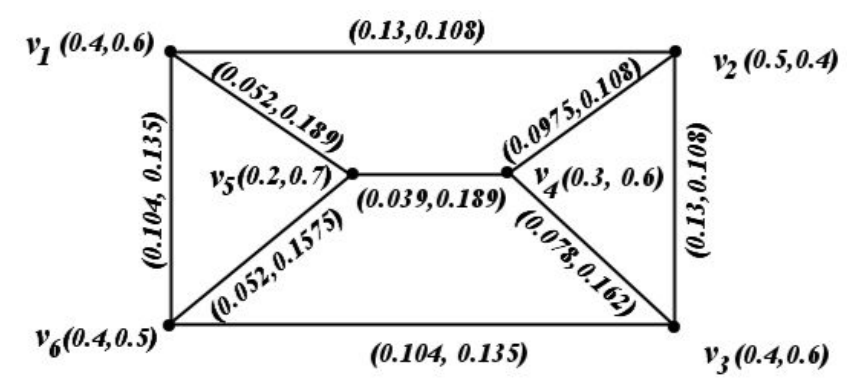

Figure 4. Product intuitionistic fuzzy graph. 
Since $G$ is strong, $\mu_{2}(u, v)=\mu_{1}(u) \cdot \mu_{1}(v)$ and $\gamma_{2}(u, v)=$ $\gamma_{1}(u) \cdot \gamma_{1}(v) \rightarrow(1)$ for every $(u, v) \in E$.

In $\bar{G}, \overline{\mu_{2}(u, v)}=\mu_{1}(u) \cdot \mu_{1}(v)-\mu_{2}(u, v)$ and $\overline{\gamma_{2}(u, v)}=$ $\gamma_{1}(u) \cdot \gamma_{1}(v)-\gamma_{2}(u, v)$.

Since $\mathrm{G}$ is strong, by (1) we have $\overline{\mu_{2}(u, v)}=0$ and $\overline{\gamma_{2}(u, v)}=0$ for every $(u, v) \in E$ and $\overline{\mu_{2}(u, v)}=\mu_{1}(u) \cdot \mu_{1}(v)$ and $\overline{\gamma_{2}(u, v)}=\gamma_{1}(u) \cdot \gamma_{1}(v)$ for every $(u, v) \in \bar{E} \Rightarrow \bar{G}$ is a strong product IFG.

Then by Corollary 3.8, $\bar{G}$ is balanced.

\subsection{THEOREM}

Every complete product IFG is a regular product IFG.

\section{ProOF:}

Let $G=(V, E)$ be a complete product IFG then by definition, the closed neighbour $\mu$ degree of each vertex is the sum of the membership values of the vertices and itself and the closed neighbour $\gamma$ degree of each vertex is the sum of the nonmembership values of the vertices and itself. Therefore all the vertices will have the same closed neighbour $\mu$ degree and closed neighbour $\gamma$ degree. This implies minimum closed neighbour degree is equal to maximum closed neighbour degree (ie) $\delta N_{\mu}[G]=\Delta N_{\mu}[G]$ and $\delta N_{\gamma}[G]=\Delta N_{\gamma}[G]$. This implies $\mathrm{G}$ is a regular product IFG.

\section{Acknowledgement}

The first author would like to thank the University Grants Commission, Hyderabad, for its financial support to Minor Research Project No. MRP-4924/14(SERO/UGC) dated March 2014.

\section{References}

1. Atanassov K. T., Intuitionistic fuzzy sets: Theory and applications, Studies in fuzziness and soft computing, Heidelberg, New York, Physica-Verl., 1999.

2. Gani A. N., and Begum S. S., "Degree, order and size in Intuitionistic fuzzy graphs", Int. J. Algorithm. Comput. Math., vol. 3(3), p. 11-16, 2010.

3. Karunambigai M. G., Akram M., Sivasankar S., and Palanivel K., "Balanced intuitionistic fuzzy graphs", Appl. Math. Sci., vol. 7(51), p. 2501-2514, 2013.

4. Kumar N. V., and Ramani G. G., "Product intuitionistic fuzzy graph”, Int. J. Comput. Appl., vol. 28(1), p. 31-33, 2011.

5. Parvathi R., and Karunambigai M. G., "Intuitionistic fuzzy graphs", Computational Intelligence, Theory and applications, International Conference in Germany, 2006.

6. Parvathi R., Karunambigai M. G., and Atanassov K., "Operations on intuitionistic fuzzy graphs", Proceedings of IEEE International Conference Fuzzy Systems (FUZZ-IEEE), p. 1396-1401, 2009. 